\title{
Spin First instructional approach to teaching quantum mechanics in sophomore level modern physics courses
}

\author{
Homeyra R. Sadaghiani and James Munteanu \\ California State Polytechnic University, Department of Physics and Astronomy, \\ 3801 W. Temple Ave., Pomona, CA, 91768
}

\begin{abstract}
As part of ongoing research in teaching and learning quantum mechanics using the Spin First approach, we are investigating student learning of basic introductory quantum concepts in sophomore level Modern Physics courses at Cal Poly Pomona in two different contexts. In one (perhaps more traditional) approach, postulates of quantum mechanics are introduced in the context of spatial wavefunctions of particles in simple potential wells; the second approach uses a Stern-Gerlach experimental context, with discrete bases of spin. Here, we compare student performances on a subset of Quantum Mechanics Concept Assessment (QMCA) posttest. We will share the results and discuss the implications for instructors of introductory quantum courses.
\end{abstract}

PACS: 01.40 . FK, 01.40.G

\section{INTRODUCTION}

The body of research examining students' understanding of quantum mechanics has been growing steadily over the past two decades. Student difficulties with learning quantum mechanics have been documented from several different perspectives. These perspectives include, but are not limited to: student difficulties with abstract concepts [1], complex mathematics, interpretation of formalisms [2], visual representations [3], and discriminating between classical and quantum phenomena [4]. Some proposed remedies to address these difficulties suggest a whole new instructional curricula design [5], whereas others are small reforms efforts to improve students' understanding of certain concepts [6]. In fact, over the past decade, substantial work has been done on quantum mechanics curriculum development both at the introductory and advanced undergraduate level [7]. In spite of vast curricula development efforts, the research on the effectiveness of these curricula is limited or in progress.

The research question this study aims to address is: how do different curricula approaches impact students' learning of different topics? More specifically, how might the use of Spin First (SF) curricula increase student performance and their ability to overcome common difficulties associated with concepts and mathematics? A study design including multiple qualitative and quantitative data collections has been crafted to address this question. In this paper, student learning of basic introductory quantum ideas in a sophomore level modern physics class using the SF approach is assessed by means of their performances on a research-based, validated diagnostic concept posttest, namely, the Quantum Mechanics Concept Assessment (QMCA) [8]. We compare student performances on the QMCA in the SF class with a Traditionally (TR) taught sophomore level modern physics and senior level quantum courses at Cal Poly Pomona (CPP).

\section{BACKGROUND}

The Spin First (SF) approach first (prior to any discussion of Schrodinger's equation) explicitly introduces the postulates of QM through the analysis of sequential Stern-Gerlach spin experiments with discrete two state spin-half bases [9]. In this approach, Dirac and bra-ket notation for states, observables, and projection operators is used from the very beginning. Students are asked to use explicit matrix representations of operators while being given opportunities to make explicit interpretations between the Dirac and matrix representations. After in-depth and detailed discussion of key quantum mechanical principles in the context of spin half, the SF approach utilizes these principles in the discussion and calculation of the wavefunction of a particle in various potential wells. This approach allows students to first focus on abstract concepts while the mathematics are simple and limited to two discrete states of spin-half.

In the Traditional (TR) approach, quantum mechanical concepts are introduced in the context of the wavefunction of a particle in various potential wells with continuous bases of position probability densities and without any explicit discussion of quantum mechanics postulates. With this approach, the focus often tends to be on carrying out mathematical processes and calculations rather than aiming for sense making based on experimental results. The postulates of quantum mechanics are not usually taught explicitly in the TR approach [10]. This approach requires students to simultaneously engage with complicated mathematical calculations and abstract concepts from the very beginning. Stern-Gerlach experiments are discussed briefly or not at all in the TR approach.

\section{A. Motivation for Spin First}

The motivation and justification for the Spin First approach has two aspects: context and mathematics. Contextually, spin is inherently quantum mechanics and forces students to break away 
from reliance on their classical mental models. In fact, researchers have found that persisting classical views can create barriers in student learning of quantum concepts [11]. Some remedies for this issue include introducing QM before classical physics, or introducing QM ideas in an unfamiliar context that does not have a classical counterpart, such as spin. Implementing the first remedy seems highly impractical. In addition, most of our classical views of the world are rooted in our everyday experiences, and not necessarily in our formal education of classical physics. However, the second remedy is a fascinating notion with some justifications for success.

Mathematically, two state spin-half is one of the simplest, yet most instructive examples with concrete experimental basis. The Spin First approach requires much simpler processes and calculations, and is limited to two-by-two matrices. Commencing with concepts based on experimental results allows students to focus on understanding rather than problem-solving performance, reducing the chance of cognitive overload. Furthermore, it seems intuitive to start with discrete bases of spin-half, upon which the more complex discussion relating to continuous bases of position probability wavefunctions can be built later in the course. For instance, the projection of the two state spin system onto its bases is represented with two histograms. Consequently, the later introduction to probability wave functions becomes a natural progression from discrete histograms to limits of continuous bases.

\section{B. Previous research}

Previous research on the effectiveness of the Spin First curriculum and the implementation of the Stern-Gerlach experiment as a teaching tool is extremely limited, but the research that does exist has shown promising results in terms of understanding and decreasing student difficulties with certain quantum mechanical concepts. For example, one study has explored the use of Stern-Gerlach experiments as a way to analyze how well students understand the differences between physical space and Hilbert space, prepare different quantum states, and differentiate between superposition states and mixtures [12]. Another one has proposed that a Spin First curriculum and computer simulations of Stern-Gerlach experiments are effective ways of pushing students towards grappling with the concepts behind the postulates of quantum mechanics, rather than simply teaching them to mechanically solve problems [13].

\section{Pilot study}

In a pilot study, we investigated the advantages of the SF method over TR means of instruction by analyzing student performance in course final examinations and class concept tests after one quarter of upper division undergraduate quantum mechanics. At CPP, the senior level quantum mechanics course is only offered once a year. Thus, the comparison of the two different approaches over two years (Fall 2011 \& Fall 2012) allowed this pilot study. In addition to quantitative data, we also administered a survey to obtain qualitative feedback from students in each course. Both courses were taught by the same instructor (HS), and were transformed from a pure lecture format to a more discussion-based format by integrating concept questions using clickers, individual and group whiteboard activities, and concept tutorials embedded in class activities.

Administering the Quantum Mechanics Concept Survey [14] pretest as a baseline for the data suggested a similar background for the two groups (55\% for SF vs. $57 \%$ for the TR group). We collected data from concept-tests, midterm and final exam questions common to both classes, and from a student survey. Although students obtained similar average scores on 34 common concept-test questions (40\% SF vs. $42 \%$ TR) during the quarter, the SF group measurably outperformed students who had received TR instruction on questions focused on the topic of quantum measurement (53\% SF vs. 33\% TR- two tail independent t-test $\mathrm{p}<$ 0.05 ). On the common midterm and final exam questions (about $40 \%$ of the exams), the SF students scored higher than the TR group. (Please see TABLE 1.)

In the class survey, we asked students about different aspects of the course such as the textbook, clicker questions, and course difficulty in terms of mathematical derivations and making sense of concepts. On one of the survey questions asking whether the mathematical aspects of the course or the concepts were more challenging, $85 \%$ of the students in the TR approach stated that the mathematical aspect of the course was more challenging than its conceptual aspects. However, in the Spin First approach, only $15 \%$ of the students found the mathematical aspect of the course more challenging than its conceptual aspect. The survey results suggest that the TR curriculum may have overwhelmed students' cognitive load with mathematical formalisms, deterring them from focusing on concepts.

TABLE 1. Common midterm and final exam scores for TR and SF group in the pilot study with the p-values for the two tail independent $t$ tests.

\begin{tabular}{l|c|c|c}
\hline & $\begin{array}{c}\text { \% Common } \\
\text { midterm }\end{array}$ & $\begin{array}{c}\text { \% Common } \\
\text { Final }\end{array}$ & $\mathrm{N}$ \\
\hline TR- Fall 2011 & $61 \pm 3$ & $64 \pm 4$ & 21 \\
\hline SF- Fall 2012 & $66 \pm 5$ & $72 \pm 3$ & 19 \\
\hline P value & 0.05 & 0.07 & \\
\hline
\end{tabular}

Although there were some indications that the SF group in the pilot study outperformed the TR group on the subset of concept questions related to quantum measurement as well as on common final exam questions, after continuing this research, it was determined that a more reliable method of ascertaining student understanding was required. This resulted in our previous work that led to the development and validation of the Quantum 
Mechanics Concept Assessment (QMCA) [8, 15]. The QMCA is a 31-item conceptually-focused survey used as a posttest to assess students' knowledge of five main topics in quantum mechanics, including, quantum measurement (Meas.), the time-independent Schrödinger equation (TISE), wavefunctions and boundary conditions (WF), time evolution (TE), and probability density (Prob.).

We are interested in learning about the impact of the SF approach on students' understanding and perception of the role of mathematics and concepts in learning quantum mechanics. Ideally, the students would not have any prior knowledge of wavefunctions, probably density, or the Schrödinger equation, however senior level students are often exposed to some introductory quantum ideas (TR approach at CPP) in their sophomore level modern physics courses. Thus, we decided to perform a study similar to the pilot study in our modern physics classes.

\section{METHODOLOGY}

Data for this study were collected from two sophomore modern physics courses at CPP, which were taught with either the SF or TR methods. In fall 2014, we used the SF approach in one section of our Modern Physics course (PHY 235, N=23) and compared student performances on a subset of QMCA questions with another class (PHY 235, N=20) that was taught in spring 2015 using the TR approach. It is worth mentioning that until fall 2014, PHY 235 was offered only once a year, during the spring quarter. However, due to increase in the number of physics majors and minors in our program, the department decided to offer the course more than once during the academic year. As a result, the fall 2014 class was an off term class, with five of the 23 students in the class repeating the course for the second time due to unsatisfactory grades in the spring 2014 class (TR approach). None of these five students scored higher than the class average on the QMCA. Hence, we felt comfortable about including their scores in the data.

We administered a fourteen-item subset of QMCA questions that were appropriate for use in sophomore level quantum courses - in class to students in both courses at the end of each quarter to measure the relative effectiveness of the two different instructional methods. The topics of this 14-item test included Meas. (7 questions), TISE (5 questions), WF (8 questions), TD (2 questions), and Prob. (4 questions). Some questions fell into more than one conceptual category. We have also compared students'

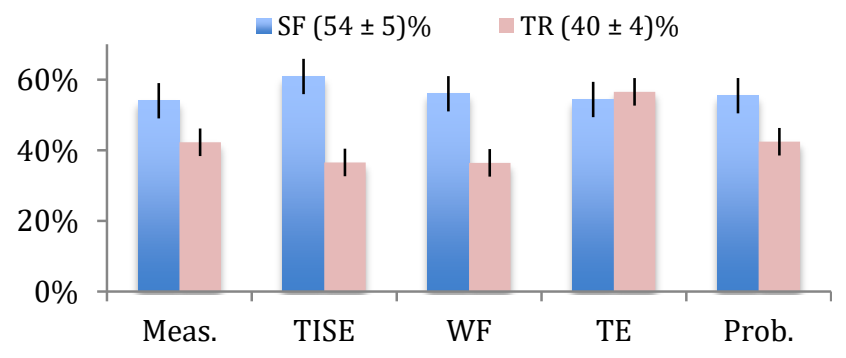

FIG 1. SF and TR modern physics students' average posttest scores on the subset of the QMCA. The error bars represent the standard error on the mean.

QMCA scores in the SF modern physics class with those in our senior level quantum course (PHY 401, N=18) that were taught in the TR format using Griffith's textbook. Using the data from the 31-item posttest QMCA that was administered to the senior level quantum class at the end of the fall 2014 quarter, we calculated student scores for the same 14 questions that were given to the modern physics class. The average for this subset of questions was used as another means of investigating the performance of the students in the SF modern physics class.

\section{RESULTS}

The average score of the students in the SF group on the subset of QMCA questions was 54\%. Comparing the SF students' overall average scores on the subset of QMCA questions with those in the TR modern physics class shows that students in the SF group outperformed the TR group by $14 \%$ (0.6 Effect size). An independent-samples t-test suggests a significant difference in the overall average scores for the two groups $(\mathrm{P}<0.05)$. The SF students' scores on all the concept categories were higher than the TR group, except for on the two questions related to Time Evolution. The details of the students' scores are illustrated in FIG 1.

Comparing the average scores of the SF students on the subset of QMCA questions with the students in our senior level course (TR Senior), we found the modern physics students did as well as advanced students who had completed one additional quarter of upper division quantum mechanics (54\% vs. $48 \%$; $\mathrm{P}>0.05$ ). (Please see FIG 2.)

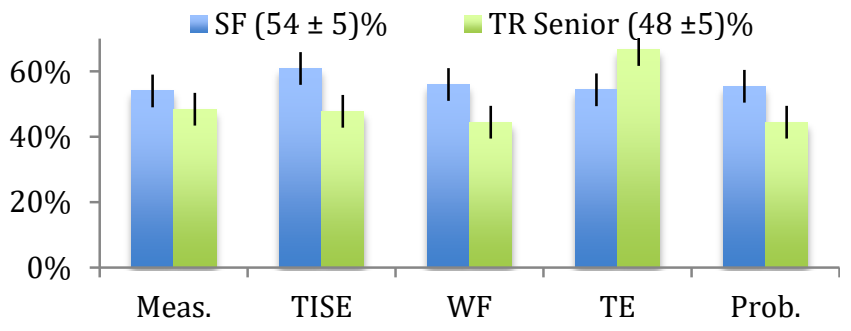

FIG 2. 14-item QMCA posttest scores for the SF modern physics course compared to the TR senior level quantum course. The error bars represent the standard errors.

The SF students scored slightly higher than their peers in the senior class on all categories except for the two questions related to TE of the wavefunction. This is not surprising, because the first few weeks of the quarter were dedicated to discussion of the historical experiments that led to development of quantum theory. Thus, topics concerning the time evolution of the wavefunction were not covered in great detail, whereas the senior level course had had more extended discussion on this topic. 
Prior to the development of the QMCA, we had been using the Quantum Mechanics Concept Survey [14] as a posttest in PHY 235 to measure student learning, which is a 12-item survey intended for use as a posttest in sophomore level courses. To see to what extent the SF approach could have affected students' learning of modern physics topics (e. g., wave-particle duality, probability wavefunctions, reflection, transmission, amplitudes and wavelengths of wavefunctions in different potentials) we administered this survey in addition to the QMCA in the SF class and compared the new results with our baseline data from past modern physics classes. FIG 3 shows that the SF students continued to outperform their peers who had experienced the TR teaching approach in previous modern physics courses $(63 \%$ vs. $57 \% \& 55 \%)$.

\section{DISCUSSION}

We are investigating the effect of the Spin First approach on the teaching of introductory quantum mechanics. This approach uses Stern-Gerlach experimental sequences and spin-half discrete bases as a context to introduce postulates of quantum mechanics. In contrast, the traditional approach often starts with introducing the Schrödinger equation and the continuous bases of position probability wavefunctions. This paper is mainly focused on a recently obtained data set for this study in which we used a 14item subset of the QMCA posttest as a means of comparing student performance in SF modern physics to the performance of students taught with the TR approach.

The survey results from the pilot study (consistent with the instructor's observation and the student feedback) indicate that the SF approach could have shifted student focus from computation to sense-making by providing concrete experimental evidence and simplifying the mathematical calculations involved. The SF approach provided students with opportunities to discuss the postulates and outcomes of quantum measurement in much more depth, independently from complex mathematical calculations.

The preliminary data shows that the Spin First approach made a positive impact on students' overall average scores on the subset of QMCA questions as compared to TR modern physics students. Although the differences reported in other the data compared in this paper (e.g., midterm and final exams, QMCS, and senior TR) are not significant, they all show a positive trend over multiple measurements. This suggests that the use of the Spin First approach not only does not hinder students' learning, it may in fact improve their understanding of certain topics.

For the next phase of this research, we are planning a longitudinal study of student learning and retention of quantum mechanical concepts. For example, in fall 2015, most students from both modern physics classes in this study (SF \& TR) will progress into a senior level quantum course. We plan to design additional surveys and interview protocols to study the likelihood of students relying on their unsuitable classical views when solving and interpreting quantum mechanics ideas. We will differentiate between students from the SF and TR groups and analyze each separately before comparing the results of the two groups.

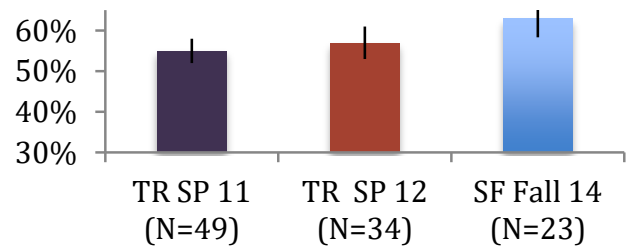

FIG 3. Posttest scores on the Quantum Mechanics Concept Survey for modern physics students in the SF \& TR classes.
[1] D. Styer, Am. J. Phys. 64, 31 (1996), C. Singh, Am. J. Phys. 69, 885 (2001). H. Sadaghiani and L. Bao, PERC Proc. AIP (2006), pp. 61-64. I. Johnston, K. Crawford, and P. Fletcher, Inter. J. Sci. Edu. 20, 427(446 (2007), A. Crouse, Ph.D. thesis, Univ. of Washington (2007).

[2] H. Sadaghiani, Ph.D. thesis, The Ohio State Univ. (2005); G. Zhu and C. Singh, Am. J. Phys. 80 p. 252 (2012).

[3] E. Çataloğlu and R. Robinett, Am. J. Phys. 70, 238 (2002).

[4] L. Bao, Ph.D. thesis, University of Maryland (1999).

[5] R. Müller and H. Wiesner, Am. J. Phys. 70, 200 (2002); D. Zollman, et. al, Am. J. Phys. 70, 252 (2002).

[6] E.g., Tutorials developed by University of Washington and Colorado on range of different quantum mechanical topics

[7] E.g., C. Singh, Am. J. Phys. 76, 400 (2008); C. Wieman, W. Adams, P. Loeblein, and K. Perkins, Phys. Teach. 48, 225 (2010).
[8] H. Sadaghiani, and S. Pollock, Phys. Rev. Spec. Top. Phys. Educ. Res. 11, 1 (2015).

[9] D. McIntyre, $1^{\text {st }}$ ed. Pearson. (2011).

[10] D. Grifiths, 3rd ed., Prentice-Hall (2003).

[11] H. Fischler and M. Lichtfeldt, Inter. J. Sci. Edu.,14, P181-190 (1992); C. Singh, 69, Am. J. Phys., 8, 885-895 (2001).

[12] G. Zhu and C. Singh, Am. J. Phys. 79, 499 (2011).

[13] C. Manogue, E. Gire, D. McIntyre, and J. Tate, PERC Procee., AIP, 55 (2012).

[14] S. McKagan, K. K. Perkins, and C. E. Wieman, Phys. Rev. Spec. Top. - Phys. Educ. Res. 6, 1 (2010).

[15] H. Sadaghiani, J. Miller, S. Pollock, and D. Rehn, PERC Proc., AIP, Portland OR (2013), p. 319. 